\title{
INCREMENTAL ALGORITHMS IN PROF-LP
}

T. Gyimóthy, T. Horváth, F. Kocsis and J. Toczki

Research Group on Theory of Automata

Hungarian Academy of Sciences

SOmogy 1 u. 7. SZEGED

H-6720 HUNGARY

\section{ABSTRACT}

PROF-LP is a programgenerator system for IBM XT/AT compatible computers. On the basis of an attribute grammar description the system generates a Pascal program which can be considered as the processor of the given specification. PROF-LP can be used for generating one-pass and multi-pass language processors. The generated one-pass processors use LL(1) method for syntactic analysis and L-attribute evaluation strategy. The multi-pass processors use LALR(1) type syntactic parser and $O A G$ attribute evaluation strategy.

The attribute grammar definition can be created in an incremental way as well. In this paper an incremental method for generating LL(1) parsing table is presented. The main advantage of the incremental system is the very fast generation of language processors.

Keywords: attribute grammars, incremental parsing, software generation

\section{INTRODUCTION}

PROF-LP (Professional Language Processor) is a programgenerator for IBM XT/AT compatible computers. The system has been developed on the basis of HLP/PAS [3], [8]. 
The input of the PROF-LP is an attribute grammar description from which a Pascal program is generated. The system is able to genexate MS-Pascal or Turbo Pascal programs.

In PROF-LP there are two ways to define an attribute grammar.

In the Batch Version [6] of the system attribute grammar description can be given in the metalanguage of the system by using a usual text editor. In the first step the system checks the correctness of the definition. After this the type of the underlying CF grammar and the class of the attribute grammar is determined by using the suitable modules of the system.

In PROF-LP there are modules for generating LL(1) and LALR(1) type parsers. The system is able to generate attribute evaluators for L-attribute [2] and OAG [5] type attribute grammars.

In the Interactive Version [7] of the system the attribute grammar definition can be defined in an incremental way. The user can give and improve the definition by using a comfortable menu system. The complete definition of the attribute grammar is a sequence of items. Each object of the grammar has an item declaring it and one or more items applying it. If an item including declarations is deleted or modified, then the other items referring to the symbols declared in it becomes "uncertain", and they are "ready" again only after reparsing. The tests and codegenerators of the system can be called by using the menu, but they do not start if one or more items are not ready.

Several large language processors have been generated by PROF-LP. The size of the generated programs were often more than twenty thousands Pascal program Iines. These large applications raised some problems in the efficient using of PROF-LP.

First of all it is very disturbing for a user, that if he makes a little modification of the large attribute 
grammar definition then the complete processor is generated and compiled again. This problem can be solved if the attribute grammar definition can be given in a modular way. So the new version of PROF-LP gives a possibility of the generation of modular compilers [8]. Unfortunately the modularity does not solve the problem of parsing table generation. If any rule in the grammar is changed then the complete parsing table of that module has to generate again. To solve this problem in the Interactive Version of PROF-LP we have developed an incremental method to test the $L L(1)$ property and to make the parsing table. Using this method it is possible to generate parsing table for large grammars in a very short time.

PROF-LP has been used to generate language processors for natural language processing and syntactic pattern recognition too. However these problems can usually be defined by ambigous grammars so the system has been extended with a backtrack parser generator. The generated backtrack parsers use the LL(1) parsing table and thus a lot of useless backtracks can be eliminated. Further characteristic of the generated parsers is that the parsing can be influenced by the evaluated attributes. Calling the procedure corresponding to the start symbol of an ambiguous grammar repeatedly the all possible derivations of a given input can be constructed [4].

In the next section of this paper the Interactive Version of PROF-LP is described. In section 3 the incremental method for LL(1) parsing table generation is presented. Some general remarks can be found in the last section.

\section{INTERACTIVE VERSION OF PROF-LP}

The Interactive Version of PROF-LP supports the incremental writing of attribute grammar definitions and on the basis of this the generation of language processors. The functions required can be chosen by the help of menu system or by direct 
typing of the commands. The textual writing of the attribute grammar definition is supported by the internal "full screen" editor. The main parts of the system are as follows:

AGDEF : definition of an attribute grammar.

GENPART: generation of a compiler.

SERVICE: service routines.

In the part AGDEF an attribute grammar can be described. So called "item"-s are used for the textual making of the syntactic-semantic and lexical description of the attribute grammar. An "item" involves an adjoining logically coherent unit of the description. The "item"-s of an attribute grammar description are as follows:

syn-decl : synthesized attribute declaration

Inh-decl : inherited attribute declaration

Nont-decl : nonterminal declaration

Token-decl : declaration of token class names

Term-decl : declaration of terminal symbols

Prod-part : production with the description of semantic activities belonging to it.

Lexs-part : lexical description.

The "item"-s are usually handled as the elements of a sequence with the exception of the "item"-s belonging to the functions Token-decl, Term-decl and Lexs-part. Because of their characteristics only one of them is necessary for the description of the given language. During the processing the state of an "item" can be the following:

Wrong: indicating that the "item" is in a syntacticallysemantically faulty state, or the "item" went wrong as a consequence of some other "item" parsing.

Complete: the "item" is faultless from syntactical

and semantical point of view.

Uncertain: the "item" got into an uncertain condition. This can happen if any other "item" has influence on this "item" and this influence does not inevitably spoil this "item".

Using the function GENPART if we have got a faultless language description (all the "item"-s should be in Complete 
state) the compiler generation can be started. The compiler generator commands to be chosen are as follows:

Finitest: the start of the module generating the finite automata of the lexical analyzer.

Lexgen: the start of the module generating the lexical analyzer.

LL(1)-test: the start of the LL(1) test and the parsing table generator module.

Codegen: the start of the codegenerator module.

Using the function SERVICE some useful service functions can be actived. These are as follows:

Profrest: it is used for the restoration of damaged language description in case of hardware error or others.

Agprint: this program provides possibility for listing the attribute grammar definition or for converting the description into "batch form".

Status: by the help of this function we can get information about the state of the attribute grammar under processing.

\section{INCREMENTAL GENERATION OF. THE LL(1) PARSING TABLE}

The Interactive Version of PROF-LP system generates LL(1) type parsers. Although the generation of these parsers is fairly fast - usually few minutes are enough for large grammars - but even a short waiting may be disturbing for an "interactive user". This waiting is especially disagreeable for a user who modified only a single rule in a large attribute gramar description. In this case an interactive system is expected to generate the suitable parser very fast. The most. time-consuming part of the $L L(1)$ parsing table generator algorithm is the computation of the FIRST 1 and Follow 1 sets. In the PROF-LP system the well-known iterative method [1] has been used to compute these sets. 
We have developed a new, incremental algorithm to the more efficient computation. The main idea of this algorithm is that the computed FIRST, and FOLLOW, sets of a grammar are always saved in a file after the processing. When this grammar is modified these sets are used in the computation of the new FIRST, and FOLLOW, sets. More exactly, the iterative algorithm starts with the "old" FIRST 1 and FOLLOW, sets except for some modified nonterminals. The initial value of those sets which can be changed with the modification of the grammar is the empty set.

In the following we give a more detail description of the incremental computation of the FIRST, and FOLLOW, sets.

Iterative formulae are given in [1] for the computing these sets. These formulae are the following: FIRST FOLLOW

$$
\begin{aligned}
& F_{0}(X)=\varnothing \\
& F_{i+1}(X)=F_{i}(X) \cup\left\{F_{i}\left(X_{1}\right) \oplus_{1} \ldots \oplus_{1} F_{i}\left(X_{n}\right)\right\}\left(x-x_{1} \ldots X_{n}\right)
\end{aligned}
$$

$$
\begin{aligned}
& H_{0}(X)=\left\{\begin{array}{ll}
\varnothing & X \neq S \\
\{\varepsilon\} & X=S
\end{array}\right\} \\
& H_{j+1}(X)=H_{j}(X) \cup\left\{F I R S T_{1}(\beta) \oplus_{1} H_{j}(Y) \mid \exists Y-\alpha X \beta\right\}
\end{aligned}
$$

In these formulae $S, \varnothing$ and $\varepsilon$ denote the start symbol, empty set and empty word respectively.

If none of the sets $F_{i}(X)$ is changed in the $i$-th step, then $\operatorname{FIRST}_{1}(X)=F_{i}(X)$ for the all nonterminals of the grammar. Similarly, FoLLOW $(X)=\mathrm{H}_{j}(\mathrm{X})$ if the sets $\mathrm{H}_{j}(\mathrm{X})$ are not changed in the $j$-th step.

Let us suppose that the FIRST FI $_{1}$ and FoLOW 1 sets of a given grammar $G$ have been computed. Denote OFIRST 1 and OFOLLOW, these sets, respectively. Let us modify the grammar $G$ and denotes $G^{*}$ the modified gramnar. Two cases are examined:

1. removing rules

2. adding new rules

The modification of a rule can be considered as the removing of the old rule and the adding of the modified one. 
Let us define the sets FID(X) and FOD(X). For the nonterminals $X$ and $Y Y \in F I D(X)$ if the FIRST, set of $Y$ "depends on" directly the FIRST, set of $x$. More exactly

$\operatorname{FID}(X)=\{(\mathrm{Y}, \mathrm{k}) \mid \exists Y \rightarrow \alpha X \beta$ with $\alpha \stackrel{*}{\rightarrow} \varepsilon\}$

where $k$ denotes the number of such rules for the nonterminal $Y$. Similarly

$\mathrm{FOD}(\mathrm{X})=\{(\mathrm{Y}, \mathrm{k}) \mid \exists \mathrm{X} \rightarrow \alpha \mathrm{X} B$ with $\beta \stackrel{*}{\rightarrow} \varepsilon\}$

The computation of the sets FID (X), FOD $(X)$ is very simply by using the sets FIRST ${ }_{1}$. (The conditions $\alpha \stackrel{*}{\rightarrow} \varepsilon, \underline{B} \stackrel{*}{\rightarrow} \varepsilon$ can easily be checked.)

Let us suppose that the sets FID, FOD have been computed for the given grammar $G$.

I. Removing of the rule $x_{0} \rightarrow x_{1} \ldots x_{n}$. Computation of the EIRST, sets

a.) Let $\operatorname{OFIRST}_{1}\left(\mathrm{X}_{0}\right)=\emptyset$.

b.) If the FIRST, set of a nonterminal $x$ directly depends on the FIRST, set of the nonterminal $\mathrm{x}_{0}$ then let $\operatorname{OFIRST}_{1}(\mathrm{X})=\varnothing$.

c.) Let us make the transitive closure of point b) by using sets FID.

d.) By using these modified OFIRST, sets as the initial sets the new FIRST, sets are computed with the original iterative algorithm. But this computation is performed only for nonterminals the OFIRST, set of which changed. Of course the number of the iterative steps in this case is also less than in case of starting from empty sets.

After the new FIRST, sets are computed a set DEC is constructed. A nonterminal $x$ is in this set if $\operatorname{FIRST}_{1}(\mathrm{X}) \subset \operatorname{OFIRST}_{1}(\mathrm{X})$.

Computation of the FOLLOW, sets

a.) A set $\mathrm{CH}$ is constructed. A nonterminal $\mathrm{X}$ is in $\mathrm{CH}$ if the set FoLLOW,$(X)$ is directly changed (or may be changed). More exactly: $\mathrm{CH}=\left\{\mathrm{X} \mid \mathrm{X}=\mathrm{x}_{\mathbf{i}}\right.$ for some $1 \leqq i \leqq \mathrm{n}$ or $\exists Y \rightarrow \alpha X \beta Z \gamma$ and $Z \in D E C$ with $\beta * \varepsilon\}$. 
b.) If $\mathrm{XECH}$ then let OFOLLOW $_{1}(\mathrm{X})=\varnothing$.

c.) If $Y \in F O D(Z)$ and the set FOLLOW $_{1}(Z)$ is modified to the empty set then let FOLLOW $_{1}(\mathrm{Y})=\varnothing$ too.

d.) Let us make the transitive closure of the point c.).

e.) By using the modified ofOLLOW, 1 sets and the new FIRST $_{1}$ sets the new FOLLOW 1 sets are computed with the original iterative algorithm.

After the sets FIRST, and FOLLOW, are computed the sets FID and FOD are modified.

$$
\text { If } x_{i} \in N(1<i \leq n) x_{1} \ldots x_{i-1}=\alpha \stackrel{\star}{\rightarrow} \varepsilon \text { and }\left(x_{0}, k\right) \in F I D\left(x_{i}\right)
$$

and $k>1$, then $\left(x_{0}, k-1\right) \in F I D\left(x_{i}\right)$ and $\left(x_{0}, k\right)$ is deleted from the set $F I D\left(X_{i}\right)$. If $k=1$ then $\left(x_{0}, 1\right)$ is deleted from the set $F I D\left(X_{i}\right)$.

If $x_{i} \in N(1 \leqq i \leqq n)$ and $x_{i+1} \cdots x_{n}=\beta \stackrel{*}{\rightarrow} \varepsilon$ and $\left(x_{i}, k\right) \in F O D\left(x_{0}\right)$ and $k>1$ then $\left(x_{i}, k-1\right) \in F O D\left(x_{0}\right)$ and $\left(x_{i}, k\right)$ is deleted from $\operatorname{FOD}\left(x_{0}\right)$. If $k=1$ then $\left(x_{i}, 1\right)$ is deleted from the set $\operatorname{FOD}\left(\mathrm{x}_{0}\right)$.

II. Adding a new rule to the grammar $G$.

This case is very simple. The initial value of the OFIRST $_{1}$ and OFOLLOW, sets of the new nonterminals is the empty set. The OFIRST, and OFOLLOW, sets of the old nonterminals are not changed. The computation of the new FID and FOD sets is also very simple, only the new dependences have to be saved.

Of course the time efficiency of this incremental algorithm strongly depends on the concrete modification of the grammar. The most time-consuming part of the algorithm is the transitive closure if the depth of the closure is large. In Figure 1 . the computation time of the sets FIRST, and FoLLOW 1 using the original iterative method is presented for two programming languages. The depth of the transitive closure in the incremental algorithm depends on the sets FID and FOD. If these sets for many nonterminals are empty or have only one element then the computation of the new FIRST, and FOLLOW, sets are very fast. 
In Figure 2. it can be seen that for example in COBOL a lot of FID sets are empty (for more than half of nonterminals) and only a few of FID sets have two or more elements.

\section{CONCLUSIONS}

In this paper the Interactive Version of the PROF-LP software generator system is described. The attribute grammar definitions of the given softwares can be defined in an incremental way. An incremental algorithm for LL(1) parsing table generation is also presented. Using this algorithm the sets FIRST, and FoLLOW, of a given grammar can be computed in a very short time.

\section{REFERENCES}

[1] Aho-Ulmann: The theory of parsing, translation and compiling, Prentice Hall, Englewood Cliffs, N. J. 1973.

[2] Bochmann, G.V.: Semantic evaluation from left to right, Comm. ACM 19. 55-62 (1976).

[3] Gyimothy at al.: An implementation of the HLP, Acta Cybernetica, 6. 3 (1983) 315-327.

[4] Gyimothy, T. and Toczki, J.: Syntactic pattern recognition in the HLP/PAS system, Acta Cybernetica 8.1. (1987) 79-88.

[5] Kastens, U.: Ordered attribute grammars, Acta Inf. 13. $229-256(1981)$

[6] PROFLP User's guide - batch version, HLP-team of the Research Group on Theory of Automata, Rep., draft, Szeged 1986.

[7] PROFLP User's guide - interactive version, HLP-team of the Research Group on Theory of Automata, Rep., draft, Szeged 1987.

[8] Toczki et al.: Automatic compiler generation, Proc. of Symp. on Automata, Languages and Pr. Systems, salgotarjan (1986). 
COBOL FORTRAN

\begin{tabular}{lcc}
\hline Number of nonterminals & 175 & 109 \\
\hline Number of terminals & 109 & 57 \\
\hline Number of syntactic rules & 377 & 202 \\
\hline Number of FIRST, iteration steps & 14 & 11 \\
\hline Number of FolLow 1 iteration steps & 14 & 13 \\
\hline FIRST, computation time & $27 \mathrm{sec}$ & $11 \mathrm{sec}$ \\
\hline FolLow, computation time & $11 \mathrm{sec}$ & $5 \mathrm{sec}$ \\
\hline
\end{tabular}

Figure 1.

\begin{tabular}{ccccc} 
& \multicolumn{2}{c}{ COBOL } & \multicolumn{2}{c}{ FORTRAN } \\
\cline { 2 - 5 } & FID & FOD & FID & FOD \\
\hline empty & 104 & 37 & 52 & 26 \\
\hline 1 & 45 & 46 & 49 & 47 \\
\hline 2 & 9 & 56 & 7 & 32 \\
\hline 3 & 12 & 24 & 1 & 1 \\
\hline more & 5 & 12 & $\emptyset$ & 3 \\
\hline
\end{tabular}

Figure 2 . 